\title{
IL17RA Gene polymorphism in Alopecia Areata in an Egyption sample E.M.Sanad ${ }^{1}$, R.M.Salem ${ }^{1}$, H.M.Ali ${ }^{2}$ and S.N.Elshafee ${ }^{1}$ \\ ${ }^{1}$ Dermatology, Venereology Dept., Faculty of Medicine, Benha Univ., Benha, Egypt \\ ${ }^{2}$ Medical Biochemistry Dept., Faculty of Medicine, Benha Univ., Benha, Egypt \\ E-Mail:shimaa22@gmail.com
}

\begin{abstract}
Alopecia areata is the demonstration of autoimmune attack against hair follicles which causes an inflammatory condition of the hair follicle. The autoimmunity is the defect in immune tolerance, resulting in the production and proliferation of autoreactive T-cells which expresses both interleukin (IL)-17A and IL-17F, both can signal via the IL-17 receptor A. To investigate the significance of IL17RA gene polymorphism in the susceptibility of alopecia areata in Egyption population. This study was carried out in Benha University, Dermatology, Venereology \& Andrology Department . We conducted a case-control association study of 100 alopecia areata patients and 100 matched healthy controls. Allele frequency of a single nucleotide polymorphim in IL17RA gene was studied using Real Time PCR technique. The A allele and AA genotype of the SNP(rs879577) of IL17RA gene incresase the risk of alopecia areata $(\mathrm{p}<0.001)$ significantly. IL17RA gene polymorphism might contribute to the increased susceptibility to alopecia areata in Egyption population.
\end{abstract}

Keywords: Alopecia areata, Interleukin-17 receptors A, Single nucleotide polymorphism.

\section{Introduction}

Alopecia areata (aa) is a chronic inflammatory disorder of hair follicle cycling owing to the collapse of the anagen-specific immune privilege with infiltration of the hair follicle by $\mathrm{T}$ lymphocytes, and characterized by nonscarring hair loss. The cause of aa is still not identified precisely; however, genetic, environmental, and immunological factors may play a role. Moreover, stress is a famous triggering and aggravating factor for the disease [1].

Consensus exists among researchers that genetic factors play an important role in the development of aa and that their identification will elucidate the underlying biological processes. Genomewide association studies (GWAS) and focused candidate gene studies have identified a total of 12 susceptibility loci for aa [2].

These include loci in the human leukocytic antigen (HLA) region, with HLA-DR being the probable key etiological factor. The availability of array-based genome wide single nucleotide polymorphism (SNP) data allows the efficient characterization of copy number variants (CNVs). These are a prominent class of genetic variants affecting larger genomic regions, which have been implicated in a number of autoimmune disorders, such as rheumatoid arthritis, Crohn's disease, systemic lupus erythematosus, psoriasis and atopic dermatitis [3].

The IL-17 family is comprised of 6 cytokines, including IL-17A to IL-17F. Among them, IL-17A and IL-17F share the highest sequence homology and have similar biological functions. Both bind to IL-17RA and IL-17RC chains. Although the genes encoding IL-17A and IL-17F are both located on chromosome 1 and 6 (respectively), in mice and humans, their functions can be similar or distinct, depending on the type of infection [4].

Interleukin-17 binds to IL-17R, which is its cognate receptor. IL-17R family consists of 5 subtypes of IL-17 receptors known as IL-17RA, IL-17RB, IL-17RC, IL17RD and IL-17RE. IL-17RA is ubiquitously expressed in tissues with a relatively higher expression in haematopoietic tissues. IL-17RB receptors are expressed in various endocrine tissues, kidney, liver and Th2 cells.
IL-17RC is expressed in non-immune cells such as prostate, thyroid and joints. IL-17RD is expressed in epithelial cells of breast, prostate, thyroid gland, ovarian surface and endothelial cells. Expression of IL-17RE is not well defined [5].

The exact role of IL-17 in the complex inflammatory cascade of aa is still not entirely clear. It is suggested that Th17 cells take the helper role together with Th1 cells to induce the inflammation around the HF, which results in aa when accompanied by a decrease in the number of $\mathrm{T}$ regulatory cells. It is speculated that the CD8+ cells then take the active role in aa progression [6].

Interleukin-17A upregulates the keratinocyte chemokine CCL20, and recruits CCR6+ cells (mDCs and Th17). Moreover, it induces the expression of neutrophil chemoattractant chemokines and antimicrobial peptides . IL-17A sustains chronic inflammations as in psoriasis [7].

\section{Aim of the work}

The aim of this work was to investigate the significance of IL17RA gene polymorphism in the susceptibility of alopecia areata in Egyption population.

\section{Subjects and methods}

- This was a case control study. The study included 100 patients suffering from different clinical presentations of Alopecia areata and 100 apparently healthy alopecia areata free individuals of matched age and sex as a control group. Patients were recurrited from the outpatient clinic of Dermatology, venereology and Andrology Department of Benha University. The study was approved by the ethics committee on research involving human subjects of Benha Faculty of Medicine. Informed consent was obtained from each individual before sample collection. aa patients were diagnosed by clinical features and physical examination including pull test . A careful history was taken from each patient and concerned general health, including the existence of 
previous aa, triggering factors, the presence of autoimmune disease, and a family history of aa or autoimmune disease Disease activity in alopecia areata patients was assessed by (SALT) score.

- Detection of IL-17RA (rs879577) polymorphism by Real Time PCR using TAQ MAN universal MMIX and TAQ MAN SNP Assay MTO HUMAN SM Kits
(Qiagen) according to manufacture instructions to detect the IL-17RA (rs879577) polymorphisms.

\section{Results}

In this study, patients and control subjects were matching regarding sex and age Table (1). Fourty four (44\%) gave history of stress as a provocative factor . History findings of the studied patients are displayed in Table (2).

Table (1) Demographic criteria of the studied groups.

\begin{tabular}{|c|c|c|}
\hline Demographic Criteria & $\begin{array}{c}\text { Cases } \\
\mathrm{N}=\mathbf{1 0 0}\end{array}$ & $\begin{array}{c}\text { Control } \\
\mathrm{N}=100\end{array}$ \\
\hline & $\mathbf{N}(\%)$ & $\mathbf{N}(\%)$ \\
\hline \multirow{3}{*}{$\begin{array}{l}\text { Male } \\
\text { Female }\end{array}$} & $39(39)$ & $35(35)$ \\
\hline & $61(61)$ & $65(65)$ \\
\hline & Range & Range \\
\hline Age (years) & $23-33$ & $22-34$ \\
\hline
\end{tabular}

Table (2) History findings in the patients .

\begin{tabular}{|c|c|c|}
\hline \multirow{2}{*}{\multicolumn{2}{|c|}{ History variables }} & Patients findings \\
\hline & & Range \\
\hline Duration (years) & & $\begin{array}{l}1-2.5 \\
\mathbf{N}(\%)\end{array}$ \\
\hline Smoking & & $28(28)$ \\
\hline $\begin{array}{l}\text { Course } \\
\text { Family History }\end{array}$ & $\begin{array}{l}\text { Stationary } \\
\text { Progressive }\end{array}$ & $\begin{array}{l}55(55) \\
45(45) \\
27(27)\end{array}$ \\
\hline
\end{tabular}

$\mathrm{N}$ : Number, SD: Standard Deviation

Multiple patches was the most common clinical presentation of the disease in the current study. Nail affection was noticed in about one quarter of the cases.

Applying Hardy Weinberg equation revealed that IL17RA genotypes and alleles frequency in patients and control groups were in Hardy Weinberg equilibrium Table (3).

Table (3) Hardy Weinberg equilibrium of Interleukin 17RA in studied patients.

\begin{tabular}{lcccc}
\hline Genotypes & \multicolumn{3}{c}{ Groups } \\
\cline { 2 - 5 } & \multicolumn{2}{c}{ Alopecia Areata } & \multicolumn{2}{c}{ Control } \\
\cline { 2 - 5 } & Observed & Expected & Observed & Expected \\
\hline GG & 12 & 11.3 & 50 & 49.1 \\
GA & 8 & 10.1 & 20 & 22.2 \\
AA & 80 & 78.6 & 30 & 28.7 \\
H.W P & & 0.059 & & 0.197 \\
\hline
\end{tabular}

\section{HW :Hardey Weinberg}

The A allele and AA genotype increase the risk of alopecia areata development significantly $(\mathrm{p}<0.001)$ Fig (1).

Relations between IL-17RA genotypes and the Studied Variables

There was non-significant relations and correlations between different IL17RA genotypes and the demographic criteria, the history findings and the clinical findings in the studied patients Tables (6-7). 


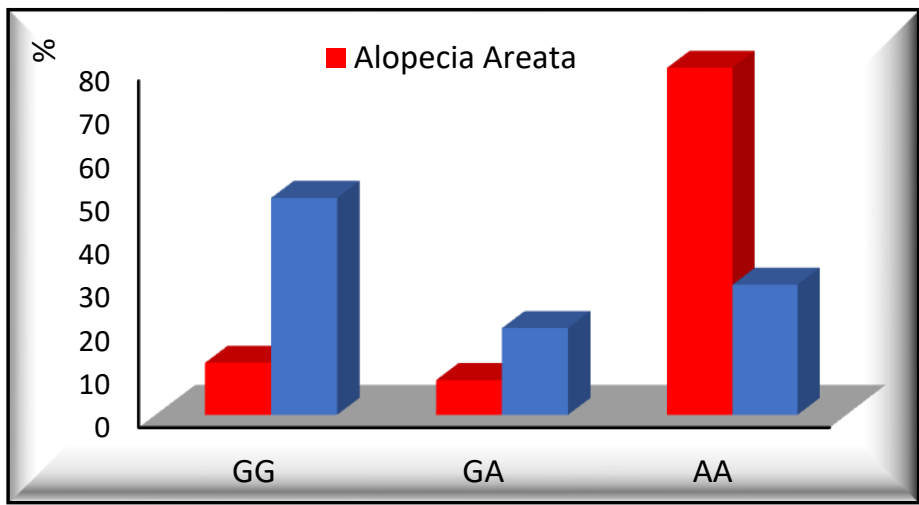

Fig (1) Interleukin17RA genotypes in the studied groups

Table (6 ) Relations between IL-17RA genotypes and the Studied Variables.

\begin{tabular}{|c|c|c|c|c|c|c|c|c|c|c|c|}
\hline & \multicolumn{8}{|c|}{ SNP (rs879577) } & \multirow{2}{*}{\multicolumn{2}{|c|}{ Chi-Square }} \\
\hline & & \multicolumn{2}{|c|}{ GG } & \multicolumn{2}{|c|}{ GA } & \multicolumn{2}{|c|}{$\mathbf{A A}$} & \multicolumn{2}{|c|}{ Total } & & \\
\hline & & $\mathbf{N}$ & $\%$ & $\mathbf{N}$ & $\%$ & $\mathbf{N}$ & $\%$ & $\mathbf{N}$ & $\%$ & $\mathbf{X}^{2}$ & P-value \\
\hline \multirow[t]{2}{*}{ Sex } & Male & 5 & 41.67 & 3 & 37.50 & 31 & 38.75 & 39 & 39.00 & 0.046 & 0.977 \\
\hline & Female & 7 & 58.33 & 5 & 62.50 & 49 & 61.25 & 61 & 61.00 & & \\
\hline Special habits & No & 9 & 75.00 & 6 & 75.00 & 57 & 71.25 & 72 & 72.00 & 0.112 & 0.946 \\
\hline of medical importance & Smoker & 3 & 25.00 & 2 & 25.00 & 23 & 28.75 & 28 & 28.00 & & \\
\hline \multirow[t]{2}{*}{ Course } & Progressive & 5 & 41.67 & 5 & 62.50 & 35 & 43.75 & 45 & 45.00 & 1.094 & 0.579 \\
\hline & Stationary & 7 & 58.33 & 3 & 37.50 & 45 & 56.25 & 55 & 55.00 & & \\
\hline \multirow[t]{2}{*}{ Family history } & Negative & 11 & 91.67 & 7 & 87.50 & 55 & 68.75 & 73 & 73.00 & 3.708 & 0.157 \\
\hline & Positive & 1 & 8.33 & 1 & 12.50 & 25 & 31.25 & 27 & 27.00 & & \\
\hline
\end{tabular}

Table (7) Correlations between Interleukin 17RA genotypes and the Studied Variables.

\begin{tabular}{|c|c|c|c|c|c|c|c|c|c|c|c|c|}
\hline \multirow[b]{3}{*}{ Age (Years) } & \multirow[b]{3}{*}{ Range } & \multirow{2}{*}{\multicolumn{6}{|c|}{$\frac{\text { SNP (rs879577) }}{\text { GA }}$}} & \multirow{2}{*}{\multicolumn{3}{|c|}{$\mathbf{A A}$}} & \multicolumn{2}{|c|}{ ANOVA } \\
\hline & & & & & & & & & & & $\mathbf{F}$ & P-value \\
\hline & & 24 & - & 37 & 21 & - & 37 & 13 & - & 40 & 0.379 & 0.686 \\
\hline Duration (Years) & Range & 0.5 & - & 5 & 0.17 & - & 3 & 0.08 & - & 4 & 0.666 & 0.516 \\
\hline
\end{tabular}

\section{Discussion}

Alopecia areata is a common, inflammatory, nonscarring type of hair loss.Significant variations in the clinical presentation of aa have been observed, ranging from small, well-circumscribed patches of hair loss to a complete absence of body and scalp hair [8].

The exact pathogenesis of aa remains unclear, but it is assumed that aa is an organ-specific autoimmune disease, mediated by autoreactive CD8+ T cells, which affects hair follicles. Atopic dermatitis, autoimmune diseases including vitiligo, and thyroid disease are associated with $\mathrm{aa}^{9}$.Interleukin-17A is the founding and the best characterized member of the IL-17 cytokine family. Overproduction of IL-17 has been associated with several chronic, autoimmune and inflammatory diseases ${ }^{10}$, however, its role in alopecia areata is not well studied yet.

The aim of this study was to study the IL17RA gene polymorphism in a sample of Egyption alopecia areata patients. The present study was conducted on a sample of Egyptian population including 100 alopecia areata patients in addition to 100 healthy control subjects of matched age and sex.

Table (8) Genetic features of the studied single nucleotide polymorphism.

\begin{tabular}{lc}
\hline Characters & The studied SNP \\
\hline ID & Rs879577 \\
Chromosomal location & 22q11.1 exon 13 \\
Gene & IL17RA \\
Alleles & G and A \\
Ancestral allele & G \\
\hline
\end{tabular}

SNP: single nucleotide polymorphism 
Both groups were in Hardy Weinberg equilibrium (HWE) regarding the studied SNP genotypes and alleles. Hardy Weinberg equilibrium excludes bias in sapmle collection ${ }^{11}$.In the present work, there was a significant link between IL17RA (rs879577) polymorphism and alopecia areata. The frequencies of IL17RA genotypes and alleles were different in alopecia areata patients and control groups. AA and GA genotypes increase the risk of alopecia areata development. This comes in agreement with ${ }^{[12]}$ who studied different IL17RA gene and found out that this SNP (rs879577) was the only one which showed significant difference between alopecia areata patients group and controls group in a Korean sample.

In agreement with ${ }^{[\mathbf{1 2}]}$, there were no significant differences of IL17RA (rs879577) genotypes in all clinical criteria of the disease in the current Egyptian aa sample. The findings of these two studies can suggest that IL17RA (rs879577) gene polymorphism can play a role in the disease development, but may not affect the clinical characters of the disease.

Treatment possibilities for aa are limited, usually do not allow sustained efficacy, and can be accompanied by significant side effects ${ }^{[13]}$. Most importantly, the lack of understanding of the underlying molecular and cytokine pathways leads to the use of broad immune antagonists, such as oral steroids or JAK inhibitors, which are efficacious, but non-specific and harbor dangerous side effects [14].

Targeting Interleukin-17A represents a novel and promising therapeutic option in a large number of autoimmune and autoinflammatory conditions. Indeed, biological drugs against this cytokine have proven effective in several disorders, including, for example, psoriasis, rheumatoid arthritis and psoriatic arthritis ${ }^{[18]}$, with a favorable safety profile. It would be beneficial to investigate these treatments in alopecia areata cases.

\section{Refrences}

[1] M .El-Gayyar, A .State, M. Helmy, E .Amer, L. Ibrahim, M .Gaballah, Evaluation of interleukin-18 and soluble interleukin-2 receptor serum levels in patients with alopecia areata: an Egyptian study. Egypt J Dermatol venereol, Vol.40, PP.34-37, 2020.

[2] J .Fischer, F .Degenhardt , A. Hofmann , Genomewide analysis of copy number variants in alopecia areata in a Central European cohort reveals association with MCHR2. Exp Dermatol, Vol.26, PP.536-541, 2017.

[3] L .Petukhova, A. Patel, R. Rigo, L. Bian, M. Verbitsky, S .Sanna-Cherchi, S. Erjavec, A .Abdelaziz, J .Cerise, A. Jabbari, A. Christiano , Integrative analysis of rare copy number variants and gene expression data in alopecia areata implicates an aetiological role for autophagy.Exp.Dermatol, Vol.00, PP.1-11, 2019.
[4] Welte, Xiang, Interleukin-17 Could Promote Breast Cancer Progression at Several Stages of the Disease .Mediators of Inflammation, Vol.2015, PP. $804347,2015$.

[5] J .Sharma, L .Balakrishnan, K. K. Datta, A. N. Sahasrabuddhe, A. Ahmad Khan, A. Sahu, A .Singhal, D .Getnet, R. Raju, A. Chatterjee, H. Gowda, K. Prasad, S. Shankar, A. Pandey , A knowledgebase resource for interleukin-17 family mediated signaling Journal of Cell Communication and Signaling, Vol.9, PP.291296, 2015.

[6] Y. Ramot, B. Marzani, D .Pinto, E. Sorbellini, F .Rinaldi , IL-17 inhibition: is it the long-awaited savior for alopecia areata? Archives of Dermatological Research; vol.310, PP.383-390, 2018.

[7] Wasilewska, Winiarska, Małgorzata, Rudnicka , Interleukin-17 inhibitors. A new era in treatment of psoriasis and other skin diseases. Dermatol Allergol;vol. (4), PP.247-252,2016.

[8] B.A. Strazzulla, , H.S.I. Eddy Chun Wang, Lorena Avila, , L.O. Kristen Sicco, , Nooshin Brinster, M. Angela Christiano, Jerry Shapiro, Alopecia areata Disease characteristics, clinical evaluation, and new perspectives on pathogenesis. J Am Acad Dermatol; vol.78, PP.1-12, 2018.

[9] You , Kim , Factors Associated with Severity of Alopecia Areata. Ann Dermatol; vol.29(5), PP.565570, 2017.

[10] N. Bernardini, N. Skroza, E. Tolino, A .Mambrin, A .Anzalone, V. Balduzzi, D. Colapietra, A .Marchesiello, S .Michelini, I. Proietti , Concetta , IL-17 and its role in inflammatory, autoimmune, and oncological skin diseases: state of art. Int.J.Dermatol; vol.5(3), PP.1-6,2019.

[11] J. Xu, A. Turner, J. Little, E.R. Bleecker , D.A. Meyers, Positive results in association studies are associated with departure from Hardy-Weinberg equilibrium: hint for genotyping error. Hum Genet; vol.111(6), PP. 573-4,2002.

[12]M.D.Lew, M.D.Hee-Ryung Cho, M.D.Sik Haw, M.D.Hwi-Jun Kim, M.D.Joo-Ho Chung, , M.D. Woo-Young Sim, the increased susceptibility to alopecia areata in Korean population, and IL17RA gene polymorphism may be associated with onset age.Ann Dermatol, Vol.24(1), PP.61-65, 2012.

[13] N.M. Luk, L.S. Chiu, K.C. Lee , Efficacy and safety of dipheny lcyclopropenone among Chinese patients with steroid resistant and extensive alopecia areata. J Eur Acad Dermatol Venereol, Vol.27, PP.400405, 2013.

[14]R. Shreberk-Hassidim, Y. Ramot, A .Zlotogorski , Janus kinase inhibitors in dermatology: a systematic review. J Am Acad Dermatol, Vol.76, PP.745$753,2017$. 